\title{
The solar coronal origin of a slowly drifting decimetric-metric pulsation structure
}

\author{
J. I. Khan ${ }^{1,2}$, N. Vilmer ${ }^{3}$, P. Saint-Hilaire ${ }^{4,5}$, and A. O. Benz ${ }^{4}$ \\ 1 Mullard Space Science Laboratory, University College London, Holmbury Saint Mary, Dorking, Surrey, \\ RH5 6NT, UK \\ 2 Stationed at: Institute of Space \& Astronautical Science, 3-1-1 Yoshinodai, Sagamihara, Kanagawa 229-8510, \\ Japan \\ 3 Observatoire de Paris, Section de Meudon, DASOP, LPSH, UMR 8645 du CNRS, 5 place Jules Janssen, \\ 92195 Meudon Cedex, France \\ 4 Institute of Astronomy, ETH Zentrum, 8092 Zürich, Switzerland \\ 5 Paul Scherrer Institute, 5232 Villigen PSI, Switzerland
}

Received 6 December 2001 / Accepted 12 March 2002

\begin{abstract}
We report observations associated with a short duration, slowly drifting decimetric-metric pulsation structure seen by the Phoenix-2 Radio Spectrometer on 2000 August 25. The range of frequencies over which this drifting radio feature occurred included frequencies observed by the Nançay Radioheliograph enabling the spatial location and development of such a radio source to be determined for the first time. The radio feature was closely associated with a solar flare. This flare was observed by the Yohkoh Soft X-ray Telescope (SXT) allowing us to compare the radio locations with the development of coronal structures seen in soft X-rays. The Yohkoh SXT images reveal two main soft X-ray features: a small flaring kernel region consisting of one or more bright loops located low in the corona and much fainter soft X-ray ejecta observed above the flare kernel region. The radio sources of the drifting pulsation structure moved outward with the soft X-ray ejecta. Our results indicate that the drifting decimetric-metric burst for this event was closely associated with the soft X-ray ejecta.
\end{abstract}

Key words. Sun: activity - Sun: corona - Sun: radio radiation - Sun: X-rays, gamma rays

\section{Introduction}

Numerous types of radio emission have been identified and classified, often according to their appearance on dynamic radio spectrograms (McLean \& Labrum 1985; Güdel \& Benz 1988; Isliker \& Benz 1994; Jiřička et al. 2001). While the origins of some types of radio bursts are generally agreed upon (e.g., type II, type III and type U bursts), there are others for which the identification is still considered speculative.

One such tentative association is the suggestion that slow drifts in the high-frequency cut-offs of decimetric pulsation structures and some other types of decimetric emission may be the radio signature of the main chromospheric evaporation process in solar flares (Aschwanden \& Benz 1995). A similar suggestion with a different scenario was proposed earlier by Karlický \& Odstrčil (1994) and later by Karlický \& Jiřička (1996) and Karlický (1998) who suggested that drifting pulsation structures seen at higher frequencies (above about $1 \mathrm{GHz}$ ) during the impulsive phase

Send offprint requests to: J. I. Khan,

e-mail: jkhan@spd.aas.org of solar flares were due to a magnetohydrodynamic shock wave ahead of evaporating chromospheric plasma.

Based on an examination of soft X-ray and microwave images during the intervals of slowly drifting decimetric pulsation features seen in radio spectrograms Hori (1999) gave another suggestion. She associated the decimetric radio emission with plasma waves excited by electron beams penetrating a prominence which is ejected in the early stages of some flares. She suggested that the observed pulsation structures at decimetric wavelengths reflect fine structures of current sheets in a reconnection region from which electron beams emanate. The drift of the decimetric radio emission was attributed to the motion and expansion of the erupting prominence, with the low and high frequency cut-offs of the radio emission associated with the upper and lower limits of the electron number density within the prominence, respectively. Similar suggestions associating drifting pulsation structures with a reconnection process involving plasmoid (loop) ejection were made independently and modeled in detail by Kliem et al. (2000).

Drifting pulsation structures (DPSs) are short duration, slowly drifting features consisting of many 
quasi-periodic pulsations. They are generally observed at decimetric wavelengths and appear to be distinct from other widely discussed slowly drifting radio emission features, such as type II bursts. For example, unlike type II bursts, DPSs only appear to occur during the impulsive phase of flares. Furthermore, there is no evidence that they exhibit harmonic emission. However, in some cases it has been suggested that DPSs may be precursors to type II bursts seen at decimetric-dekametric wavelengths (Karlický \& Odstrčil 1994; Karlický \& Jiřička 1996). Whether DPSs are, in some sense, similar to the type II precursors reported by Klassen et al. (1999) is not yet clear. The causal relation, if any, between DPSs and subsequent type II bursts remains an open issue.

Recent explanations for DPSs favor an association with a reconnection scenario associated with so-called plasmoid ejection (e.g., Karlický et al. 2001), similar to the suggestions of Hori (1999) and Kliem et al. (2000), rather than with the chromospheric evaporation process.

Kundu et al. (2001) reported metric-decimetric radio emission above and after two flare-associated soft X-ray plasmoid ejection events. They presented no spectral data in their paper. But one event was identified as a stationary radio type IV burst, while the radio spectral classification of the other event was not given.

Recently, Hudson et al. (2001) identified a rapidly moving hard X-ray source seen with the Yohkoh Hard X-ray Telescope (HXT) (Kosugi et al. 1991) during the impulsive phase of a solar flare. This was associated with a rapidly moving microwave source and inferred to be associated with a plasmoid (loop) ejection. The rapidly moving sources appeared to occur during the time of a high frequency slowly drifting pulsation structure. However no radio images of the DPS emission are available. There are also no soft X-ray images of their event.

In this paper we present observations associated with a slowly drifting decimetric-metric pulsation structure seen by the Phoenix-2 Radio Spectrometer (Messmer et al. 1999) during the impulsive phase of a solar flare which occurred on 2000 August 25. We use Nançay Radioheliograph (NRH) (Kerdraon \& Delouis 1997) data to identify the spatial location and development of this type of radio emission for the first time. The radio sources are compared with soft X-ray images from the Soft X-ray Telescope (SXT) (Tsuneta et al. 1991) on board the Yohkoh satellite (Ogawara et al. 1991) to identify the associated coronal structures.

\section{Observations}

In Fig. 1 we present a dynamic radio spectrogram from the Phoenix-2 Radio Spectrometer showing a decimetricmetric feature on 2000 August 25. It appeared to consist of at least two components, a group of type III bursts from about 14:29-14:31 UT (which extended from $280 \mathrm{MHz}$ to below $220 \mathrm{MHz}$ ) and a slowly drifting feature from about 14:29:30-14:33:30 UT (which extended above $550 \mathrm{MHz}$ to below $220 \mathrm{MHz}$ ). The drifting radio feature consisted of several tens of pulsations. The frequency drift rate of the feature, as determined from cross-correlation analysis, was $-2.8 \pm 0.5 \mathrm{MHz} \mathrm{s}^{-1}$ at a mean frequency of $430 \mathrm{MHz}$. This drift is at the upper limit of the range observed in type II bursts (Nelson \& Melrose 1985). If this drift is interpreted as due to an exciter moving in a stationary atmosphere with a temperature of $2 \mathrm{MK}$, then the drift rate would suggest the speed of the exciter was about $1300 \mathrm{~km} \mathrm{~s}^{-1}$.

The DPS was associated with a flare of X-ray class M1.4 and optical class 1N, which began at about 14:23 UT on 2000 August 25 in NOAA sunspot region 9143. The heliographic coordinates of the center of the soft X-ray flare were roughly S16E68. The soft X-ray flare as seen with the low energy $(\sim 1-8 \AA)$ channel of the GOES 8 satellite is shown in Fig. 2. Superimposed on this plot are hatched areas representing the time intervals of Yohkoh satellite night. Also presented in Fig. 2 is the Yohkoh HXT L ( 14$23 \mathrm{keV}$ ) channel flux, which shows clearly the interval of the impulsive phase of the flare. The interval of the DPS which extended from 14:29:30-14:33:30 UT is also indicated on the plot.

Prior to the flare, Yohkoh SXT observations consisted of full-frame images (FFIs) of the whole Sun and high time cadence (quiet-mode) partial-frame images (PFIs) of a different active region (NOAA region 9131 on the west limb). From 14:27:42 UT until just before satellite night Yohkoh was in flare-mode. During that interval there were no FFIs, only high time cadence (flare-mode) PFIs of the flaring region. Hard X-ray data is also avaliable in the three other channels of the HXT. We very briefly discuss some of the Yohkoh HXT data later. In this paper we emphasize comparisons of the Yohkoh SXT data with the radio data for this event.

Superimposed in Fig. 2 are small vertical tick marks indicating the times of the Yohkoh SXT data near the time of the radio event. The flare-mode PFIs consist of $128 \times 128$ full resolution $(2.45$ arcsec) pixel images taken with the $\mathrm{Al}$ $0.1265 \mu \mathrm{m}$ (henceforth Al.1) and AlMgMn soft X-ray filters (Tsuneta et al. 1991). Early in the flare, as the time cadence of the PFIs was high, the tick marks have the appearance of a black band in Fig. 2. The Al.1 flare-mode PFIs for this event were all long exposure images which over-exposed the flare kernel region on the SXT CCD, but which showed the evolution of fainter features at higher altitudes. The AlMgMn images during most of the impulsive phase of the flare had their exposure values adjusted to minimize the saturation in the SXT CCD, thereby showing the flare kernel, but not the fainter features at higher altitudes.

The radio feature overlapped the 236.6, 327.0, 410.5 and $432.0 \mathrm{MHz}$ frequencies of the Nançay Radioheliograph. Thus we are able to directly determine the source location of a drifting pulsation structure for the first time. In Figs. 3a,b we show the whole-Sun fluxes measured at each of the relevant NRH frequencies. The DPS corresponds to the broad, mound-shaped features seen in the latter part of the fluxes at 236.6 and $327.0 \mathrm{MHz}$. We will discuss Figs. 3c-f later. 


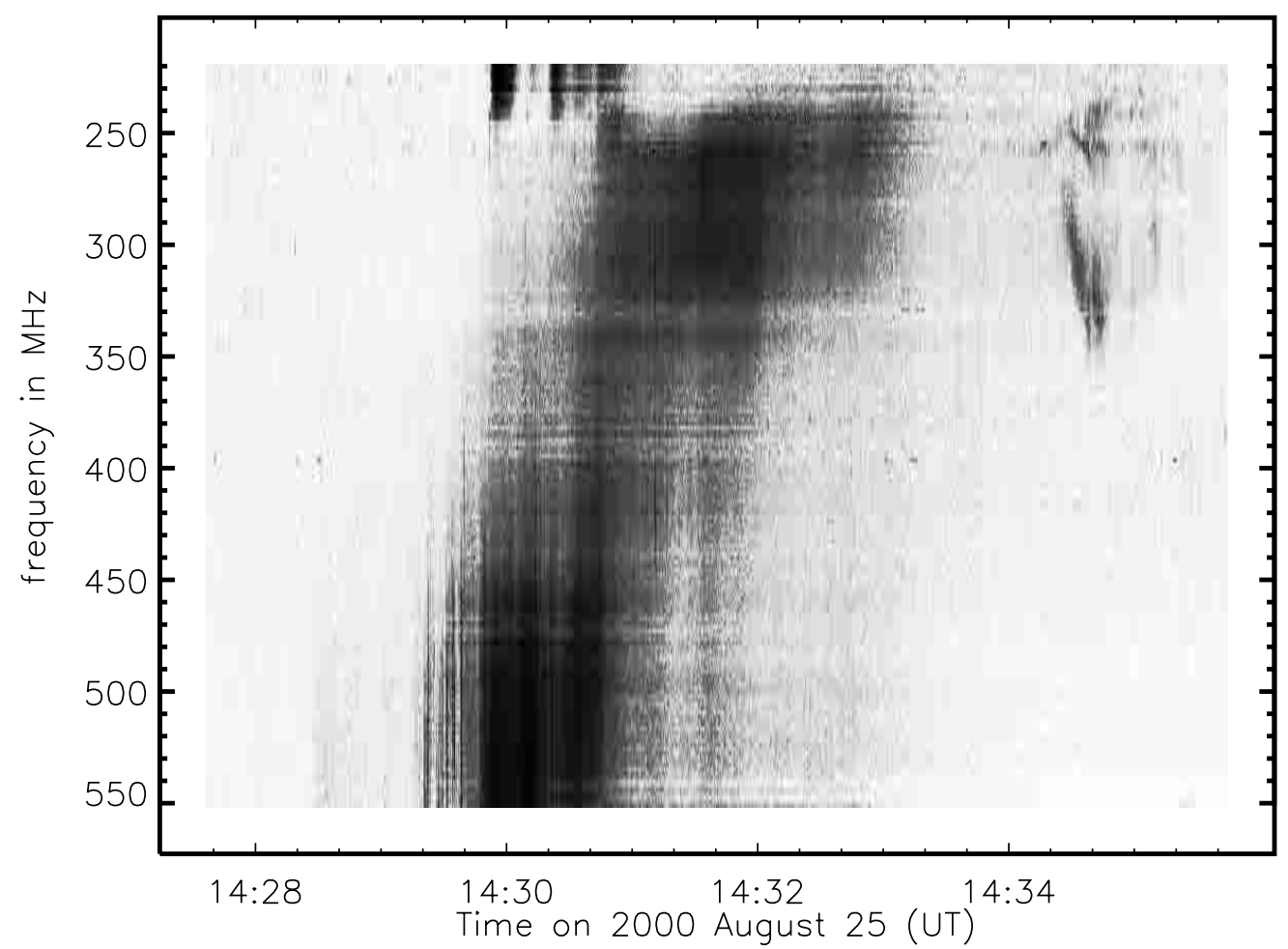

Fig. 1. Phoenix-2 radio spectrogram showing a slowly drifting decimetric-metric pulsation structure on 2000 August 25 . The white horizontal lines are caused by calibration errors.

In Fig. 4a we show an example of a portion of an NRH $327.0 \mathrm{MHz}$ image during the DPS. The $\mathrm{NRH}$ image is displayed using a reverse linear black and white color table (where darker denotes higher intensity). In Fig. 4b we show an SXT Al.1 PFI at 14:30:54.4 UT closest to the time of the NRH 327.0 MHz image in Fig. 4a. The SXT image is also displayed using a reverse black and white color table, but the SXT images have been scaled considering only a narrow range of normalized output values of the pixels, taking the square-root, and then linearly scaling the values to span the full range of display values. This scaling exaggerates the apparent brightness of faint features making them easier to see. The black vertical strip in the Al.1 PFI shows the region of saturated pixels on the SXT CCD due to the flare. Superimposed on this saturated region is a portion of the AlMgMn PFI taken at 14:30:46.4 UT showing the bright flaring kernel. On this composite SXT image we overplot the contours for the NRH $327.0 \mathrm{MHz}$ source shown in Fig. 4a. This example shows an apparent soft X-ray mass ejection which we loosely term a plasmoid close to the location of the DPS. By the term plasmoid we mean an apparent blob-like feature probably due to a magnetic flux loop. Since the plasmoid was observed only with the Al.1 filter it is not possible to measure its temperature and emission measure using the SXT filter ratio technique. Hence it is not possible to estimate the particle number density of the plasmoid for this event to check whether this agrees with the range in the particle number density of the DPS determined from its range in radio frequency. Note also that the shape of the NRH contours are suggestive of a loop structure.

As mentioned earlier, during the relevant Yohkoh satellite orbit, there were no SXT PFIs in the vicinity of the flaring region until 14:27:42 UT. Comparison of that image with the next Al.1 image at 14:27:58 UT reveals motion of the plasmoid as shown in Fig. 5. Consequently, the plamoid eruption began before 14:27:58 UT, either sometime during the interval between 14:27:42-14:27:58 UT or earlier.

In Fig. 6 we show a sequence of portions of Al.1 PFIs from 14:27:58-14:32:30 UT. The Al.1 images shown in Fig. 6 were displayed by first truncating each image over different ranges for each image, then taking the square root and finally linearly scaling each image to span the full range of display values. This scaling was done to show the soft X-ray mass ejection as clearly as possible in each image. Note that due to this scaling the actual relative differences in brightness between the images cannot be compared directly from this figure. All saturated pixels were set to zero and appear white in Fig. 6. In some of the images even parts of the soft X-ray mass ejection were saturated on the SXT CCD. Superimposed on some of these images are portions of AlMgMn PFIs (nearest in time to the SXT Al.1 PFIs) showing the flare kernel region. The scaling of the AlMgMn images is similar to that of the Al.1 images, except that the pixel values were not truncated prior to scaling. Dotted black lines bisecting each edge of 


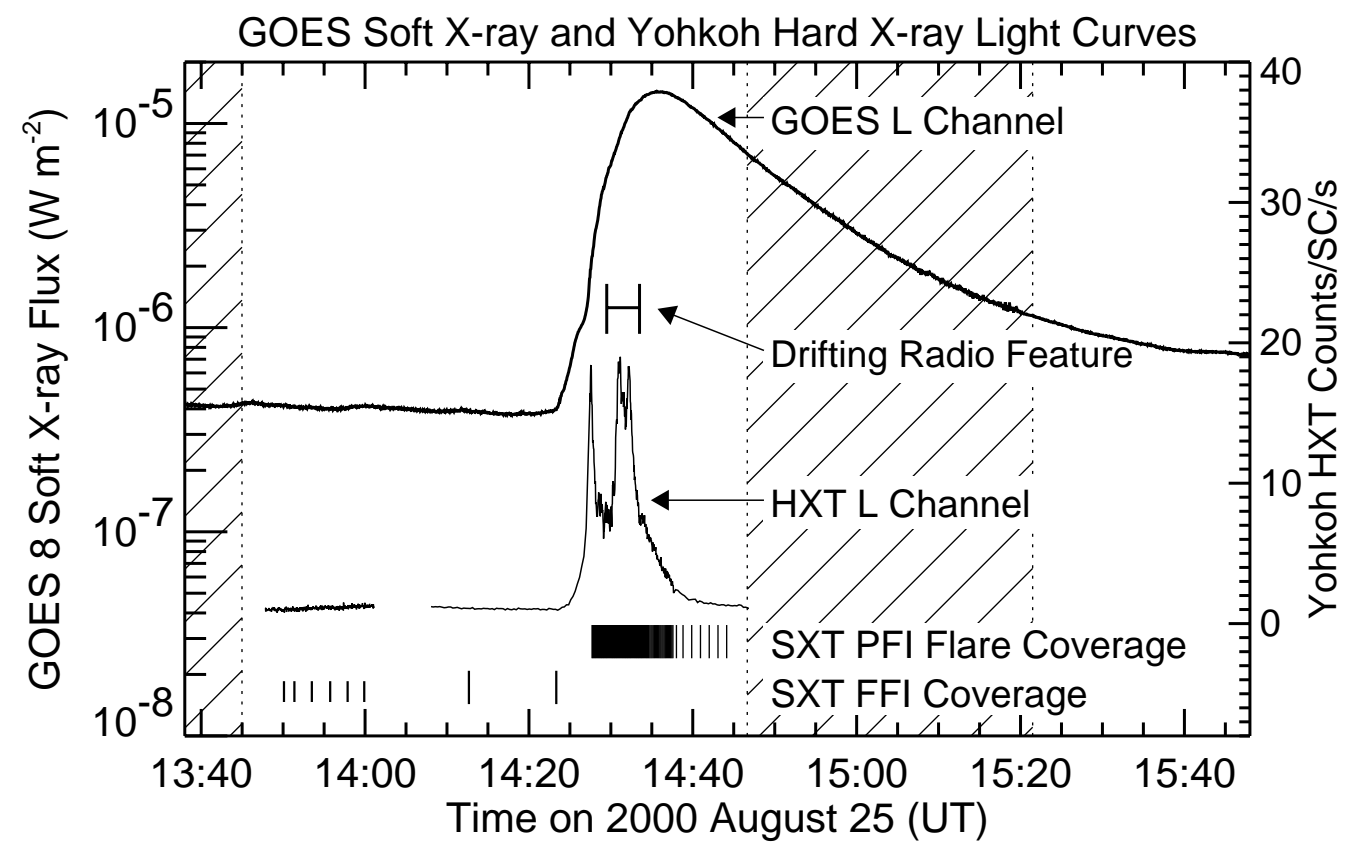

Fig. 2. The soft X-ray flux for the low energy $(\sim 1-8 \AA)$ channel from the GOES 8 satellite showing the soft X-ray flare associated with the DPS on 2000 August 25. Also shown is the observed HXT L $(\sim 14-23 \mathrm{keV})$ channel count rate per subcollimator. The times of SXT full-frame images (FFIs) and flare-mode partial-frame images (PFIs) are indicated by small vertical tick marks. The hatched time intervals indicate the time intervals of Yohkoh satellite night.

the image portions have been drawn to help the reader see the apparent motion of the soft X-ray features. These SXT images indicate the ejection of soft X-ray emitting plasma above the flare kernel (some parts of the ejection are indicated by white arrows in Fig. 6). We also show NRH 327.0 MHz images (as 90\% contour levels displayed with thick solid black lines) nearest in time to the SXT Al.1 PFIs. At the top of each panel in Fig. 6 we show the times of the Al.1 PFIs, while at the bottom we give the times of the NRH 327.0 MHz images. The times of the Al.1 PFIs in Figs. 6h-o are indicated with ticks marks at the bottom of the flux plots of Figs. 3a,b. The DPS was seen at $327.0 \mathrm{MHz}$ at all of these times as is apparent from Fig. 3a. The SXT and NRH 327.0 MHz data in Fig. 6 indicate that the DPS emission occurred near a soft X-ray plasmoid and that the DPS emission moved outward away from the flaring kernel roughly with the soft X-ray plasmoid.

We fitted a 2-D elliptical Gaussian function to each of the NRH images. The shape of the NRH sources of the DPS were generally not close to the shape of a 2-D elliptical Gaussian function, as is apparent from Fig. 4. The aim of the fitting was to estimate the approximate locations of the centroids of the NRH sources and hence determine general trends in the motions of those sources. Consequently, we applied the fits only to pixels within $60 \%$ of the peak value in each image. We show the $x$ - and $y$-coordinates (in NRH pixel units) of the centroid locations of the fitted sources in Figs. 3c-f. The technique we used appeared to adequately describe the actual source centroids for the sets of 236.6 and $327.0 \mathrm{MHz}$ source locations shown in Figs. 3c and e. The 236.6 MHz source locations shown are for the DPS and the preceding type III bursts. The 410.5 and $432.0 \mathrm{MHz}$ sources often showed complex or multiple sources. Thus the locations of those sources shown in Figs. 3d and f are approximate only. We divide the interval of the DPS seen at $327.0 \mathrm{MHz}$ into two intervals. The $x$-coordinates of the fits to the 236.6 and $327.0 \mathrm{MHz}$ sources show generally decreasing values (indicating eastward motion). The $y$-coordinates of the fits to the 236. $6 \mathrm{MHz}$ sources show decreasing values (indicating southward motion). The $y$-coordinates of the fits to the $327.0 \mathrm{MHz}$ sources show first an interval of increasing values followed by an interval of decreasing values. Linear fits to the $x$ - and $y$-coordinates of the fits to the 236.6 and $327.0 \mathrm{MHz}$ sources can be used to estimate the speeds of the radio sources. Above each of the intervals of the linear fits in Figs. 3c and e we give the speeds (in $\mathrm{km} \mathrm{s}^{-1}$ ) implied by the linear fits. From these speeds of the co-ordinates we find that the projected speed of the source of the drifting pulsation structure seen at $236.6 \mathrm{MHz}$ was $257 \mathrm{~km} \mathrm{~s}^{-1}$, while the speeds at $327.0 \mathrm{MHz}$ were (in sequential order) 438 and $291 \mathrm{~km} \mathrm{~s}^{-1}$.

It is difficult to estimate the speed of the soft X-ray plasmoid seen in the SXT images reliably due to the change in brightness of the soft X-ray emitting features with time and the lack of a clearly distinct feature to track. Nonetheless we attempted to track the plasmoid and determine its speed from a linear fit to the height-time plot of the points. Our best estimate is that the speed of the soft X-ray plasmoid seen in projection in the SXT images was $290 \pm 60 \mathrm{~km} \mathrm{~s}^{-1}$ over the interval 14:27:42-14:30:06 UT. Thus the projected speed of the soft X-ray plasmoid is similar to the projected speeds of the sources of the DPS 

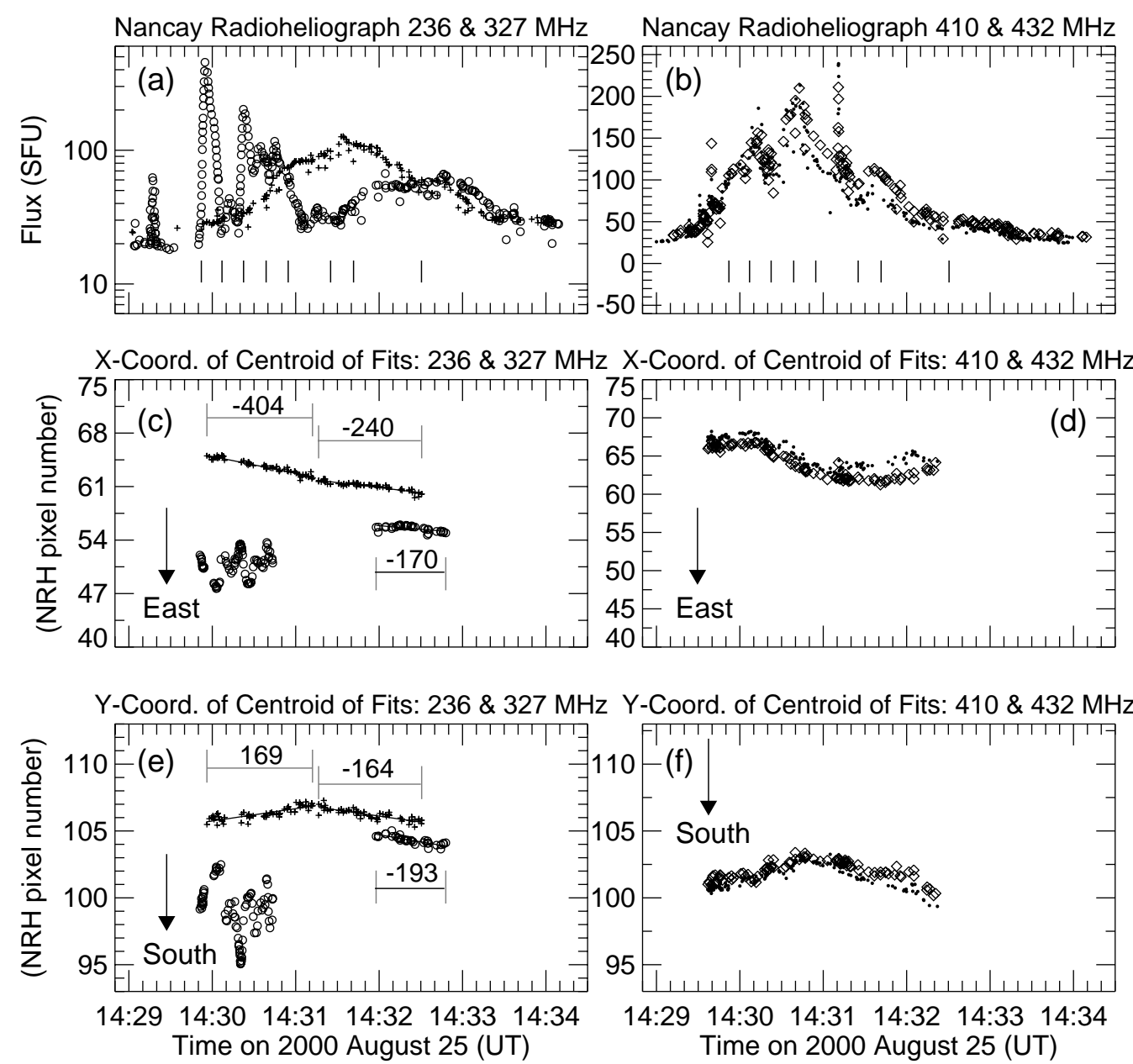

Fig. 3. a) The Nançay Radioheliograph ( $\mathrm{NRH})$ whole-Sun fluxes at $236.6 \mathrm{MHz}(\circ)$ and $327.0 \mathrm{MHz}(+)$ and b) at $410.5 \mathrm{MHz}(\diamond)$ and $432.0 \mathrm{MHz}(\bullet)$. Also shown at the bottom of $\mathbf{a})$ and $\mathbf{b}$ ) are tick marks indicating the times of SXT images which will be discussed later. Panels $\mathbf{c}$ ) and $\mathbf{d}$ ) show the $x$ (east-west) positions (in units of NRH image pixels) of the centroids of 2-D elliptical Gaussian fits to the NRH radio sources (using the same symbols for each frequency), while e) and f) show the $y$ (north-south) positions. Also shown in c) and $\mathbf{d}$ ) are the ranges over which linear fits to the positions were made. Above these intervals we give the speed (in $\mathrm{km} \mathrm{s}^{-1}$ ) implied by the linear fits for each coordinate. The best fit lines are also overplotted.

at 236.6 and 327.0 MHz. That the speed of the soft X-ray plasmoid was similar to the speed of the $\mathrm{NRH} 327.0 \mathrm{MHz}$ source could also be inferred from the relative separation of the radio and soft X-ray sources with time, as seen in Fig. 6.

In Fig. 7 we consider the last eight panels of the SXT Al.1 images in Fig. 6, i.e., Figs. 6h-o, but we present a wider field of view to show the other radio sources. Instead of showing the AlMgMn images of the flare kernel as in Fig. 6 we place portions of the HXT L $(\sim 14-23 \mathrm{keV})$ channel images nearest in time to the SXT images over the saturated SXT pixel regions. We have examined the data in all four HXT channels and find the hard X-ray sources for this flare were all located near the flaring kernels. We do not find any hard X-ray emission in the vicinity of the plasmoid ejection. The times shown in each panel in Fig. 7 are of the Al.1 PFIs (these correspond to the tick marks shown in Figs. 3a,b). On these SXT images we show the locations of the radio sources at 236.6, 327.0, 410.5 and $432.0 \mathrm{MHz}$ (as $90 \%$ contour levels) nearest to the Al.1 PFIs.
We have already discussed the $327.0 \mathrm{MHz}$ sources. We now briefly discuss the radio sources at the other $\mathrm{NRH}$ frequencies. From Fig. 3a the 236.6 MHz sources seen in Figs. $7 \mathrm{f}-\mathrm{h}$ are of the DPS. These appeared to lie directly above the $327.0 \mathrm{MHz}$ sources of the DPS. From Figs. 3c and e it can be seen that during the initial interval when the DPS was observed at both 236.6 and $327.0 \mathrm{MHz}$, i.e., 14:31:57-14:32:30 UT, the 236.6 and $327.0 \mathrm{MHz}$ sources of the DPS moved away from the flaring region with similar speeds and maintained an approximately fixed separation. The 236.6 MHz images in Figs. 7a-e are of the type III bursts which were located significantly further away from the flaring region and the soft X-ray plasmoid (see also Figs. 3c and e). Distant location of type III bursts has been noted previously by others (e.g., Poquerusse et al. 1988). From Fig. 7 and Figs. 3d and f the 410.5 and $432.0 \mathrm{MHz}$ radio sources showed outward motions roughly similar in magnitude and direction to the 236.6 and $327.0 \mathrm{MHz}$ sources of the DPS. However, while the $432.0 \mathrm{MHz}$ source were located approximately below the $410.5 \mathrm{MHz}$ source 

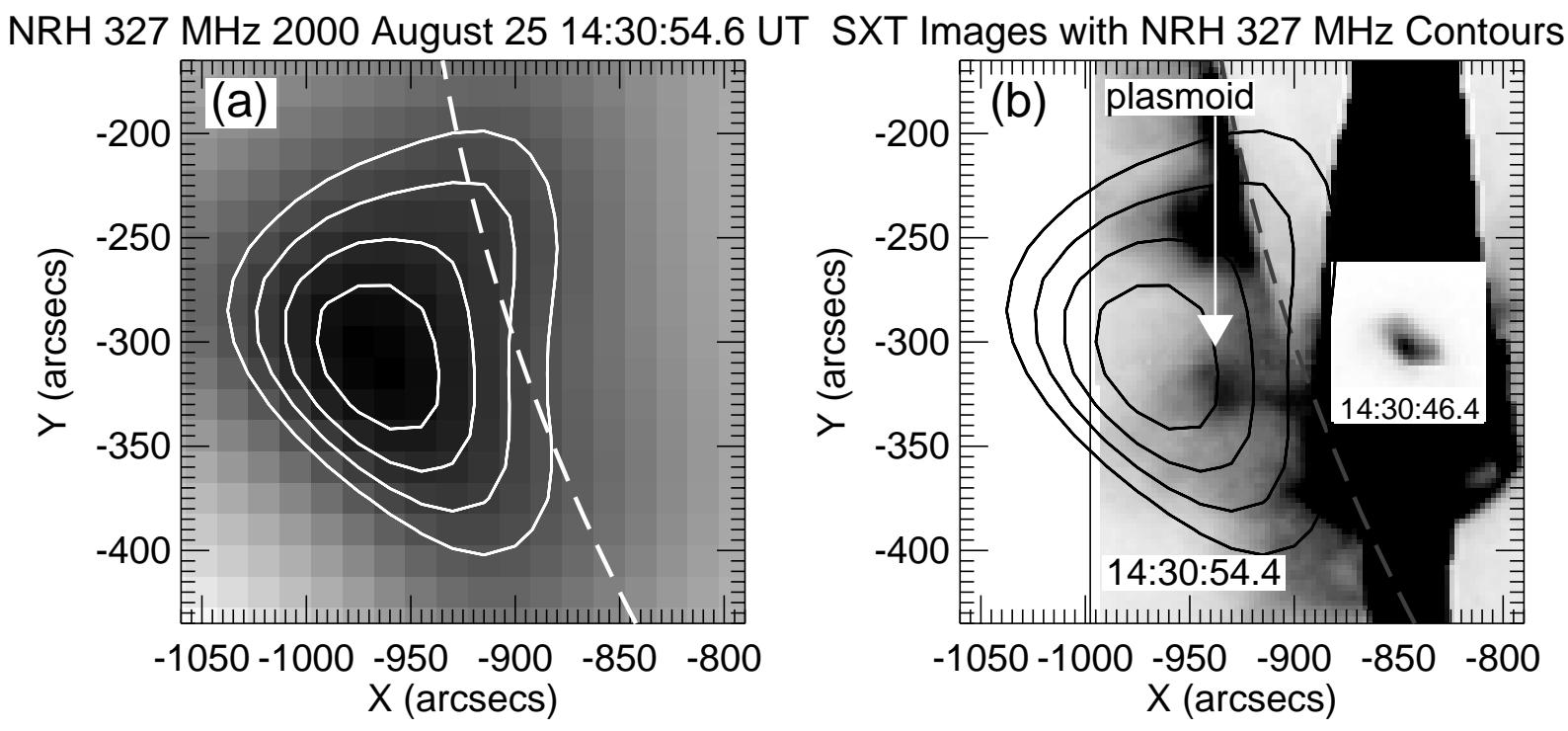

Fig. 4. a) An image from the NRH showing the source of the 327.0 MHz emission at 14:30:54.6 UT, during the DPS, (with superposed contours $(60 \%, 70 \%, 80 \%, 90 \%$ of the peak of the image) of the same NRH source). b) A composite SXT image with the NRH source contours shown in a) overplotted. The solar limb is indicated by the dashed line in each image.
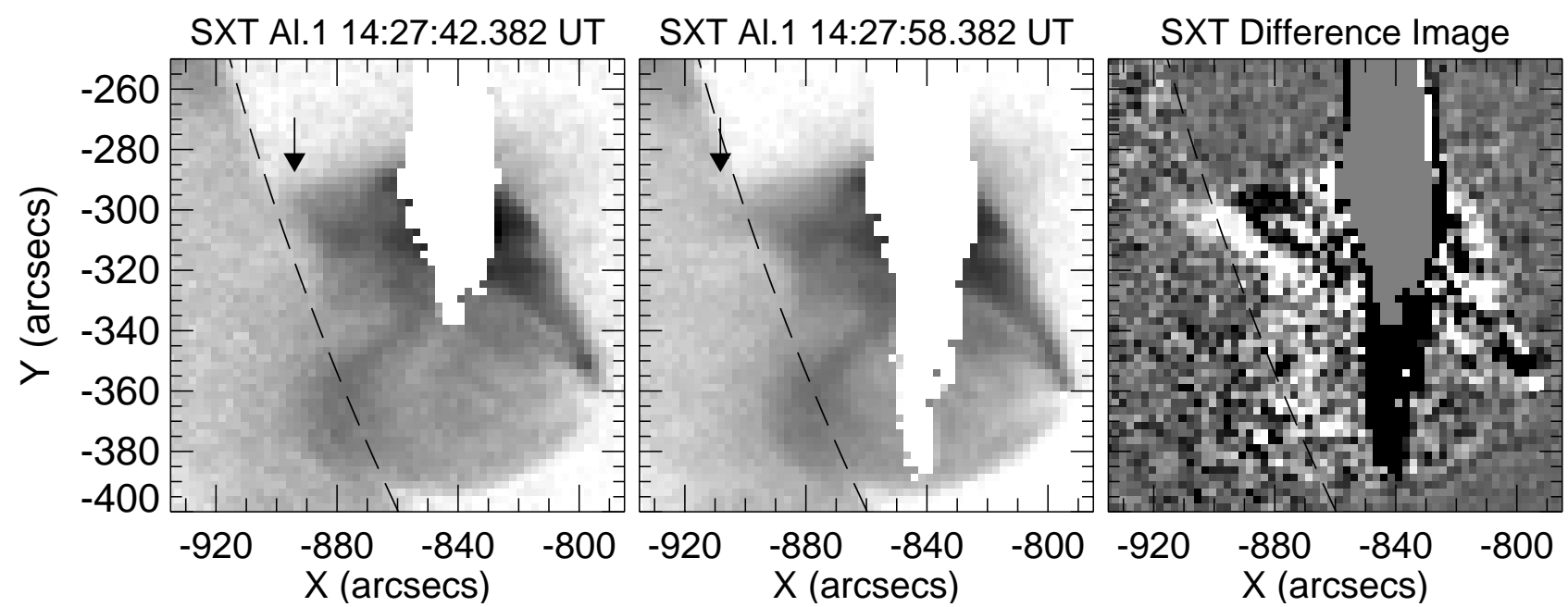

Fig. 5. The first two images are the earliest SXT Al.1 images of the flaring region (scaled logarithmically and to show the relative intensities between them). The saturated pixels were set to zero and appear white in those images. These images show that motion of the soft X-ray plasmoid was observed early in the flare. The last image is the difference of the first image minus the second image scaled over an appropriate range to show the moving features. In each image the dashed black line indicates the solar limb.

at each time shown in Fig. 7 (see also Figs. 3d and f), the 410.5 and $432.0 \mathrm{MHz}$ DPS sources were adjacent to, not below, the $327.0 \mathrm{MHz}$ DPS sources. That is, they did not lie between the flaring kernel and the sources of the DPS at 327.0 or $236.6 \mathrm{MHz}$. The good temporal correlation of the individual pulsations, as seen in the radio spectrogram, between 200-550 MHz (Fig. 1) indicates that the emission from each of the individual pulsations were from magnetically connected structures.

Although it is beyond the scope of the present paper to discuss in detail propagation effects on the apparent relative source locations of the DPS, some brief comments are in order. Refraction would move sources to locations of apparently higher density, while scattering by plasma inhomogeneities and ducting effects in magnetic flux tubes would move sources to locations of apparently lower density (and/or increase the size of the sources). While such effects will undoubtedly take place, they are difficult to estimate reliably. At times when the DPS was seen at both 236.6 and $327.0 \mathrm{MHz}$ the locations of those radio sources agree roughly with what is expected if they originated from a structure where the density gradient was responsible for the apparent spatial differences between the sources. The lack of alignment between the lower (236.6 and 327.0 MHz) frequency sources and the higher $(410.5$ and $432.0 \mathrm{MHz})$ frequency sources suggests that the difference in position for those sources is not due 


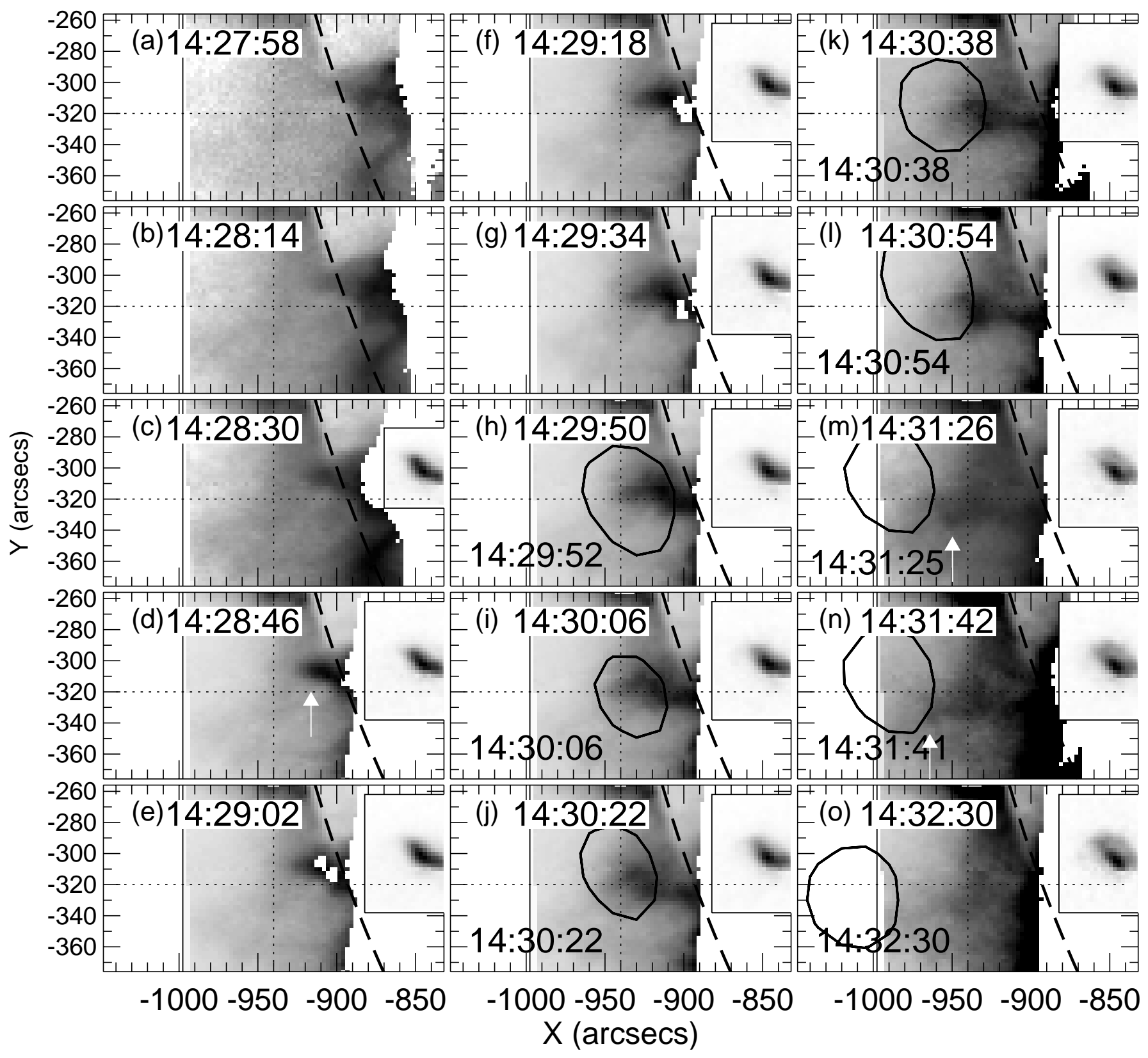

Fig. 6. A sequence of SXT (composite) images showing the evolution of the soft X-ray ejecta with the NRH $327.0 \mathrm{MHz}$ sources nearby in time (indicated by the $90 \%$ contour level in each radio image) prior to and during the drifting pulsation structure. The solar limb is indicated by the thick dashed black line in each image.

to propagation effects. Rather it may be due to sources originating from somewhat different features.

\section{Conclusions}

In this paper we have presented the first analysis of radio imaging observations of a drifting pulsation structure seen at decimetric-metric wavelengths. We examined the soft X-ray images associated with the DPS and find that the site of the radio emission was not near the bright soft X-ray flaring kernel. Such a location would be expected for an association with chromospheric evaporation, since the main chromospheric evaporation is expected to have occurred in those loops which later appeared very bright in soft X-rays and which also had hard X-ray emitting footpoints. Clearly the DPS was not associated with the main chromospheric evaporation process in the flare.

Images taken at overlapping time intervals show that the sources of the drifting pulsation structure were located close to a soft X-ray emitting mass ejection seen above the flaring loops during the impulsive phase of the flare. The speed of the DPS observed in the NRH $327.0 \mathrm{MHz}$ images and early in the $236.6 \mathrm{MHz}$ images was found to be broadly similar to the speed of the soft X-ray emitting plasmoid inferred from the SXT images.

The soft X-ray mass ejection, which we refer to loosely as a plasmoid, appeared blob-like in the SXT images 


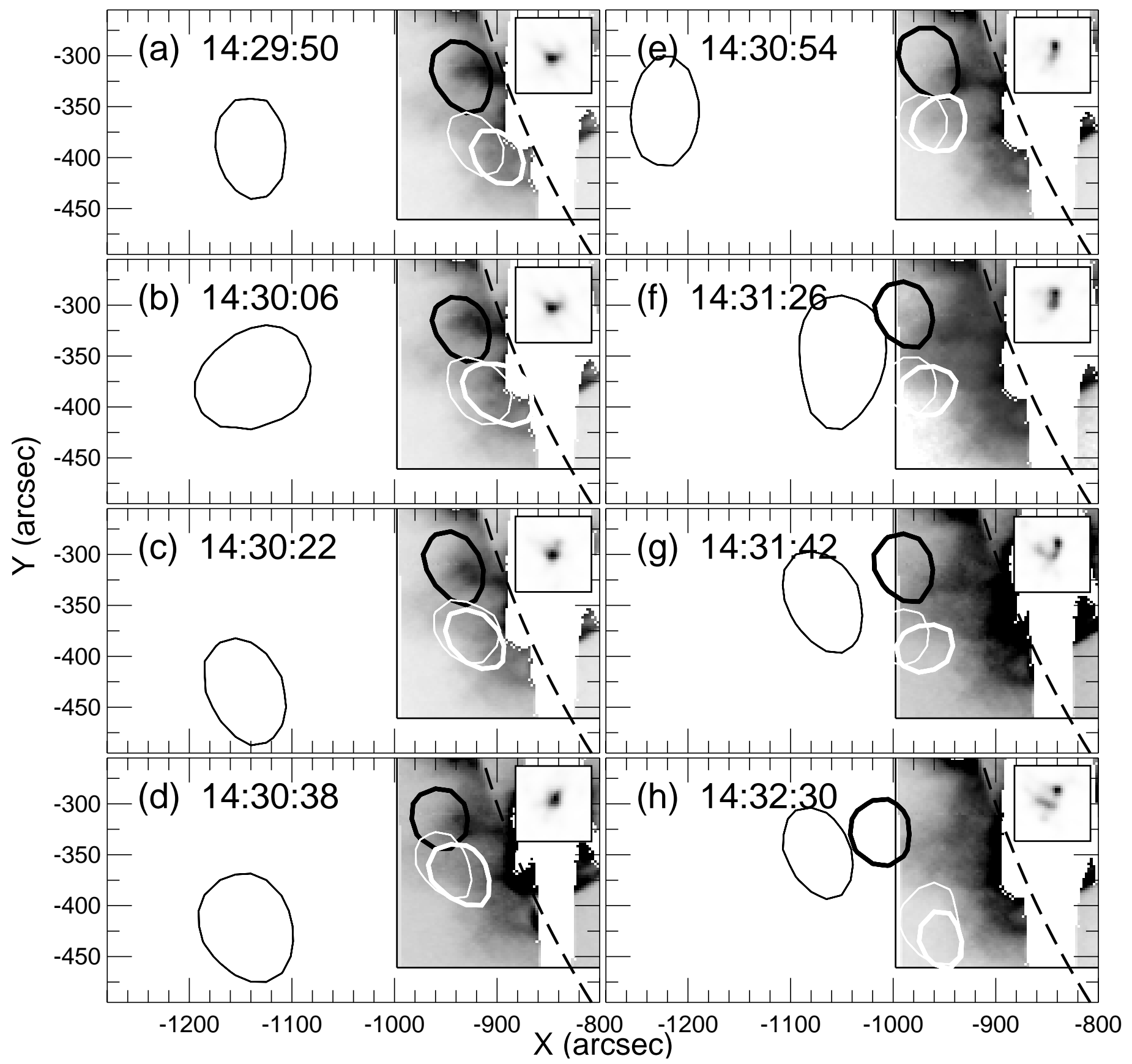

Fig. 7. A sequence of SXT Al.1 images showing the evolution of the soft X-ray features and the locations of the NRH sources (as indicated by the $90 \%$ contour levels): $236.6 \mathrm{MHz}$ (thin black), $327.0 \mathrm{MHz}$ (thick black), $410.5 \mathrm{MHz}$ (thin white) and $432.0 \mathrm{MHz}$ (thick white). Also shown in the boxed region over the saturated pixel region of the SXT images are HXT L ( 14-23 keV) channel images nearest in time to the SXT images.

and somewhat similar to the soft X-ray ejecta reported by Shibata et al. (1995) and Ohyama \& Shibata (1997, 1998). The SXT images show that the blob-like soft X-ray emitting region was connected to lower regions (via soft $\mathrm{X}$-ray emitting structures) indicating that the plasmoid was probably part of a loop structure or bundle of loops. The shape of the contours of the NRH 327.0 MHz sources of the DPS suggests the DPS may have originated from a loop-like structure in the vicinity of the soft X-ray plasmoid. Thus our results provide clear observational support for the suggestions made by Hori (1999), Kliem et al. (2000) and Karlický et al. (2001) that drifting pulsation structures are closely associated with the process of plasmoid ejection during the impulsive phase of solar flares.

The observations presented here also enable us to discern the nature of the probable association of the DPS with the plasmoid. If the radio emission of a DPS is related to the plasma frequency, then the negative drift implies decreasing electron number density with time: $\frac{\mathrm{d} \nu}{\mathrm{d} t}=\frac{\mathrm{d} \nu}{\mathrm{d} n} \frac{\mathrm{d} n}{\mathrm{~d} t}$, 
where $\nu$ is the frequency, $n$ is the electron number density and $t$ the time.

The decreasing electron number density could be interpreted in terms of the velocity of a radio exciter moving through an isothermal solar atmosphere with an exponential decrease in the density. The negative drift rate would correspond to the velocity $v$, with which the exciter propagates through the atmosphere. This is the usual interpretation for type II bursts. In such a scenario, the emission at each frequency corresponds to a specific height in the atmosphere, with lower frequencies at greater heights. Note that for a near-limb event, as examined in this paper, the line of sight may be nearly orthogonal to the density gradient. If so, for the case of a moving exciter, no significant outward drift of the radio source locations at a given frequency would necessarily be expected.

An alternative interpretation would be in terms of an expanding plasmoid of radius $R$. In that scenario the drift rate then amounts to $\frac{\mathrm{d} \nu}{\mathrm{d} t}=-\frac{3}{2} \frac{\nu}{R} \frac{\mathrm{d} R}{\mathrm{~d} t}$. If the plasmoid expands due to outward motion, then the source locations at each frequency would also show outward motion.

Since the NRH sources of the DPS at each frequency showed outward motion away from the flaring region we infer that the source of the radio emission occurred not at a fixed height in the solar atmosphere, but was associated with an emitting region which moved outward through the solar atmosphere. We find that when the DPS was seen at both 236.6 and $327.0 \mathrm{MHz}$ the locations of those sources maintained a roughly similar relative separation as they moved outward away from the flaring region. This indicates that the DPS emission was directly related to the ambient particle density in the vicinity of the moving plasmoid.

The speed $\left(1300 \mathrm{~km} \mathrm{~s}^{-1}\right)$ inferred from the drift of the DPS seen in the radio spectrogram is much greater than the SXT- and NRH-derived speeds. However, that speed was derived under the assumption of an exciter propagating in a stationary atmosphere, which we reject. We associate the observed frequency drift of the emission in the spectrogram with the decreasing density in the plasmoid, not the speed of propagation of an exciter moving through a stationary atmosphere.

We find that the ejection of the soft X-ray plasmoid was observed prior to the detection of the DPS. This indicates that the radio emission or its exciter may be due to the build up of some effect caused by the motion of the plasmoid (such as reconnection cutting off magnetic field lines behind the plasmoid).

It has been suggested (e.g., Kliem et al. 2000; Karlický et al. 2001), that the DPS emission might originate from (i) a reconnection region between an ejected plasmoid and underlying loops, (ii) locations where a reconnection outflow jet are stopped (i.e., above underlying loops or below an overlying ejected plasmoid), or (iii) from inside or in the vicinity of a plasmoid (i.e., from particles threading through or trapped within the magnetic structure containing the plasmoid).
Our observations indicate that the source locations of the DPS at $327.0 \mathrm{MHz}$ were roughly aligned with either the center or upper parts of the soft X-ray plasmoid, while those at 236.6 MHz were located at higher altitudes (outside the SXT Al.1 PFI field of view). Although the size of the radio source, say as indicated by the $60 \%$ contour levels seen in Fig. 4, was large and included a wide region surrounding the erupting plasmoid structure, we can make some tentative conclusions as to which of the proposals (i)-(iii) may be the most consistent with the present observations. It appears that the source locations of the DPS at 236.6 MHz may occur at or above the inferred outer edge of the soft X-ray plasmoid (comparing with earlier images when the DPS was seen only at $327.0 \mathrm{MHz}$ ). The development (i.e., outward motion) and, in particular, the observed locations, of the 236.6 and $327.0 \mathrm{MHz}$ sources appear to exclude proposals (i) and (ii). Proposal (iii) appears to be the most consistent with the present observations.

The temporal overlap of the DPS with part of the hard $\mathrm{X}$-ray bursts indicates that both the radio and hard X-ray emission features were associated with particles accelerated during the impulsive phase of the flare. The DPS was due to upward moving particles, while the hard $\mathrm{X}$-ray bursts were due to downward moving particles. The soft X-ray and radio imaging observations presented here indicate that the DPS originated from particles as they passed through or became trapped within the magnetic structure containing the plasmoid. We conclude that the spectral drift of the drifting pulsation structure was due to the expansion of the plasmoid as it propagated away from the flaring region.

It appears likely that events of the type examined by Hudson et al. (2001) may be similar to the type of the event examined in this paper. The results presented in this paper confirm the conclusions of Kundu et al. (2001) that metric-decimetric radio emission is associated with the propagation of a soft X-ray plasmoid. Moreover, we have identified a particular type of radio feature associated with the process, at least for our event, namely, a slowly drifting pulsation structure. Two other examples of plasmoids (temporally) associated with slowly drifting pulsation structures were given by Hori (1999) and Kliem et al. (2000). Whether plasmoid ejections are commonly associated with slowly drifting pulsation structures, or vice-versa, is not yet clear.

Acknowledgements. The Yohkoh mission is a project of the Institute of Space and Astronautical Science (ISAS) of Japan. It is financially supported by ISAS, NASA, and the Particle Physics and Astronomy Research Council (PPARC) of the UK. The Nançay Radio Observatory is funded by the French Ministry of Education, the Centre National de la Recherche Scientifique (CNRS), and the Région Centre. The construction of the Phoenix-2 Radio Spectrometer is supported by the Swiss NSF (Grant No. 2000-061559). The GOES data are courtesy of the World Data Center A for Solar-Terrestrial Physics, NGDC, NOAA E/GC2, 325 Broadway, Boulder, Colorado 80303, USA. J.I.K. received support from PPARC. 


\section{References}

Aschwanden, M. J., \& Benz, A. O. 1995, ApJ, 438, 997

Benz, A. O., Bernold, T. E. X., \& Dennis, B. R. 1983, ApJ, 271,355

Güdel, M., \& Benz, A. O. 1988, A\&AS, 75, 243

Hori, K. 1999, in Solar Physics with Radio Observations, Proceedings of the Nobeyama Symp., NRO Report No. 479, 267

Hudson, H. S., Kosugi, T., Nitta, N. V., \& Shimojo, M. 2001, ApJ, 561, L211

Isliker, H., \& Benz, A. O. 1994, A\&AS, 104, 145

Jiřička, K., Karlický, M., Mészárosová, H., \& Snížek, V. 2001, A\&A, 375, 243

Karlický, M. 1998, A\&A, 338, 1084

Karlický, M., \& Jiřička, K. 1996, Sol. Phys., 163, 171

Karlický, M., \& Odstrčil, D. 1994, Sol. Phys., 155, 171

Karlický, M., Yan, Y., Fu, Q., et al. 2001, A\&A, 369, 1104

Kerdraon, A., \& Delouis, J.-M. 1997, in Coronal Physics from Radio and Space Observations, ed. G. Trottet (Berlin: Springer-Verlag), Lect. Notes in Phys., 483, 192

Klassen, A., Aurass, H., Klein, K.-L., Hofmann, A., \& Mann, G. 1999, A\&A, 343, 287
Kliem, B., Karlický, M., \& Benz, A. O. 2000, A\&A, 360, 715

Kosugi, T., Makishima, K., Murakami, T., et al. 1991, Sol. Phys., 136, 17

Kundu, M. R., Nindos, A., Vilmer, N., et al. 2001, ApJ, 559, 443

McLean, D. J., \& Labrum, N. R. (ed.) 1985, Solar Radiophysics (Cambridge: Cambridge University Press)

Nelson, G. J., \& Melrose, D. B. 1985, in Solar Radiophysics, ed. D. J. McLean, \& N. R. Labrum (Cambridge: Cambridge University Press), 333

Messmer, P., Benz, A. O., \& Monstein, C. 1999, Sol. Phys., 187,335

Ogawara, Y., Takano, T., Kato, T., et al. 1991, Sol. Phys., 136,1

Ohyama, M., \& Shibata, K. 1997, PASJ, 49, 249

Ohyama, M., \& Shibata, K. 1998, ApJ, 499, 934

Poquerusse, M., Steinberg, J. L., Caroubalos, C., Dulk, G. A., \& MacQueen, R. M. 1988, A\&A, 192, 323

Shibata, K., Masuda, S., Shimojo, M., et al. 1995, ApJ, 451, L83

Tsuneta, S., Acton, L. W., Bruner, M. E., et al. 1991, Sol. Phys., 136, 37 